\title{
Distributed Model-Based Fault Diagnosis with Stochastic Uncertainties
}

\author{
Francesca Boem and Thomas Parisini
}

\begin{abstract}
This paper proposes a novel distributed fault detection and isolation approach for the monitoring of non linear large-scale systems. The proposed architecture considers stochastic characterization of the measurement noises and modeling uncertainties, computing at each step stochastic timevarying thresholds with guaranteed false alarms probability levels. The convergence properties of the distributed estimation are demonstrated. A novel fault isolation method is proposed basing on a Generalized Observer Scheme, providing guaranteed error probabilities of the fault exclusion task. A consensus approach is used for the estimation of variables shared among more than one subsystem; a method is proposed to define the time-varying consensus weights in order to minimize at each step the variance of the uncertainty of the fault detection and isolation thresholds. Detectability and isolability conditions are provided.
\end{abstract}

\section{INTRODUCTION}

In this paper, we propose a distributed model-based Fault Detection and Isolation (FDI) approach with stochastic bounds on the measurement noise and modeling uncertainty, deriving probabilistic thresholds for the fault detection and isolation residuals. The presented architecture is specifically designed for the monitoring of large-scale, networked, distributed systems. These systems are characterized by a large number of states and inputs and are typically spatially distributed. The interest towards Large-Scale Systems (LSSs) (see, for example, [1]), Systems-of-Systems [2] and CyberPhysical Systems [3], and their reliability requirements, is steadily growing both in industry and academia. When monitoring this kind of systems, the design of distributed or decentralized methods is usually necessary due to computational, communication, scalability and reliability limits (see [4], [5], [6], [7], [8], [9], [10],[11], as examples). We model these systems as a network of many subsystems connected through physical or communication interactions. Differently from previous works ([7], [8], [12]) where a deterministic approach was adopted, in this paper the novelty is to consider stochastic bounds on the noises and uncertainties. The aim is to propose a monitoring architecture which is closer to industrial applications, where deterministic bounds on the uncertainties are often difficult to be obtained, producing then conservative results.

It is worth noting that the proposed technique is not a data-driven method ([13] for a recent survey), but a modelbased one [14] with stochastic uncertainties, which uses the

This work has been conducted as part of the research project Stability and Control of Power Networks with Energy Storage (STABLE-NET) which is funded by the RCUK Energy Programme (contract no: EP/L014343/1).

F. Boem is with the Dept. of Electrical and Electronic Engineering at the Imperial College London, UK. (f . boemeimperial.ac.uk)

T. Parisini is with the Dept. of Electrical and Electronic Engineering at the Imperial College London, UK, and with the Dept. of Engineering and Architecture at University of Trieste, Italy. (t.parisini@gmail.com) knowledge of the model of the system in order to compute some local state estimates and related detection and isolation thresholds. An integration of data-driven and model-based approaches is proposed in [15], but in a centralized scenario, while here both detection and isolation are performed in a distributed way.

A similar distributed fault detection architecture has been introduced in [16] which is dealing with the design of an optimal decomposition of the LSS and the fault isolation is not addressed. In this paper, as novel contribution and differently from [16], we provide detectability results, we prove the convergence of the estimation error mean and we define a novel time-varying consensus approach for the estimation of state variables shared among more than one subsystems, and thus monitored by more than one local diagnosers. Moreover, we propose a method to analytically compute the consensus weights to minimize the amplitude of the thresholds, minimizing the variance of the uncertainty. Furthermore, here we consider also the fault isolation problem, guaranteeing a certain probability error level. The results on fault isolability show the conditions on the local fault function so that the monitoring system can exclude the non-occurring faults. The derived fault detection and fault isolation thresholds are stochastic and able to guarantee a certain false alarms probability. The distance of the residual from the isolation thresholds is characterized, allowing to obtain less conservative results.

The problem formulation and some of the results that we provide here about fault detection, are similarly introduced in [17]. While in this paper the system topology is fixed and our concern is in developing fault detectability and isolability analysis and in proposing a novel Fault Isolation architecture, [17] aims at investigating the proposed Fault Detection scheme for LSS with a time-varying topology. To the authors" knowledge, this is the first time that a model-based fault isolation architecture is presented in the distributed scenario taking into account the stochastic features of measurement noise and modeling uncertainties. The developed method can be extended also in a Plug-and-Play scenario (following the approach presented in [17]).

\section{PROBLEM FORMULATION}

Let us consider a LSS, composed of $M$ interconnected subsystems. The discrete-time dynamics of each subsystem can be described as

$$
\Sigma_{[i]}: x_{[i]}^{+}=f_{i}\left(x_{[i]}, \psi_{[i]}, u_{[i]}\right)+w_{i}(t)+\phi_{i}\left(x_{[i]}, \psi_{[i]}, u_{[i]}, t\right)
$$

where $x_{[i]} \in \mathbb{R}^{n_{i}}, u_{[i]} \in \mathbb{R}^{m_{i}}, i \in \mathcal{M}=\{1, \ldots, M\}$, represent the local state and local input vectors, respectively, at time $t$ and $x_{[i]}^{+}$denotes $x_{[i]}$ at time $t+1$. The intercon- 
nection variables vector $\psi_{[i]} \in \mathbb{R}^{p_{i}}$ collects the components of $\left\{x_{[j]}\right\}_{j \in \mathcal{N}_{i}}$ that influence the dynamics of $x_{[i]}$, where $\mathcal{N}_{i}$ is the set of the parents of subsystem $i$. It is defined as $\mathcal{N}_{i}=\left\{j \in \mathcal{M}: \frac{\partial x_{[i]}^{+}}{\partial x_{[j]}} \neq 0, i \neq j\right\}$. We also introduce $\mathcal{C}_{i}=\left\{k: i \in \mathcal{N}_{k}\right\}$ as the set of children of $\Sigma_{[i]}$. We say that $\Sigma_{[i]}$ and $\Sigma_{[j]}$ are neighbors if $j \in \mathcal{N}_{i}$ or $j \in \mathcal{C}_{i}$.

Let us provide more insight about the model in (1): $f_{i}(\cdot): \mathbb{R}^{n_{i}} \times \mathbb{R}^{p_{i}} \times \mathbb{R}^{m_{i}} \rightarrow \mathbb{R}^{n_{i}}$ describes possibly nonlinear local nominal dynamics, including known relationships with parent subsystems by means of the interconnection variables, while $w_{i}(\cdot): \mathbb{N} \rightarrow \mathbb{R}^{n_{i}}$ represents modeling uncertainties and disturbances, considering unknown coupling among subsystems. Finally, the fault function $\phi_{i}(\cdot): \mathbb{R}^{n_{i}} \times \mathbb{R}^{p_{i}} \times$ $\mathbb{R}^{m_{i}} \times \mathbb{R} \rightarrow \mathbb{R}^{n_{i}}$, which is null before the unknown fault time $T_{0}$, models deviations of the dynamics of $\Sigma_{i}$ from the nominal healthy dynamics. The $k$-th component of vector $x_{[i]}$ is denoted by $x_{[i, k]}$. In this paper, we assume that the state vector is fully accessible through possibly noisy measurements. The local output equation is $y_{[i]}=x_{[i]}+\varrho_{[i]}$, where $\varrho_{[i]} \in \mathbb{R}^{n_{i}}, i \in \mathcal{M}$, is the local unknown measurement error at time $t$. Similarly, $z_{[i]}=\psi_{[i]}+\theta_{[i]}$ is the vector of measured interconnection variables communicated by father subsystems, with $\theta_{[i]}$ collecting the involved measurement error $\varrho_{[j]}, j \in \mathcal{N}_{i}$.

The following assumptions are needed:

Assumption 1: The modeling uncertainty $w_{i}$ is a unknown function, modeled as a stochastic process with unknown distribution. We assume to know at each time instant $t$ the mean and the variance of the stochastic variables $w_{i}(t)$, for all $i \in \mathcal{M}$ :

$$
w_{i}(t) \approx\left(\mu_{w_{i}}(t), \sigma_{w_{i}}(t)\right) .
$$

Assumption 2: The measurement noise $\varrho_{[i]}$ is a stochastic process of known distribution. We assume to know at each time instant $t$ the mean and the variance of the stochastic variables $\varrho_{[i]}(t)$ for all $i \in \mathcal{M}$ :

$$
\varrho_{[i]}(t) \approx\left(\mu_{\varrho_{[i]}}(t), \sigma_{\varrho_{[i]}}(t)\right) .
$$

Each subsystem is monitored by a local agent, called Local Fault Diagnoser (LFD). The considered decomposition of the LSS is overlapping [1] since some of the variables "belong" to more than one subsystem. In fact, some state variables, which we call shared variables, are monitored by more than one LFD (see Fig.1).

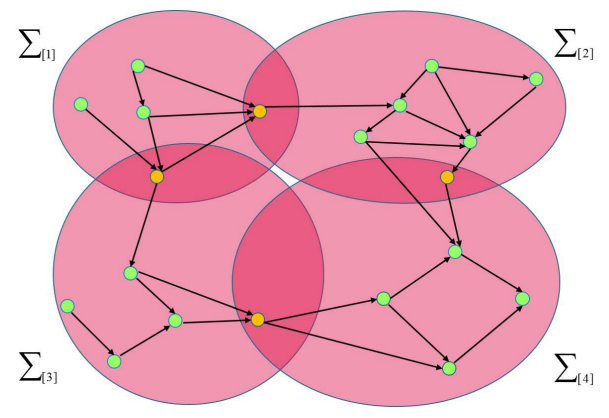

Fig. 1. The possibly overlapping decomposition of the LSS structural graph: the small green circles represent the state and input variables; the yellow ones are the shared state variables.

\section{The Fault Detection Architecture}

In this section, we design a distributed FD architecture in a stochastic framework. An estimate $\hat{x}_{[i]}$ of the local state variables is defined; the estimation error $\epsilon_{[i]} \triangleq y_{[i]}-$ $\hat{x}_{[i]}$ is then compared component-wise with some properly designed time-varying stochastic detection thresholds $\bar{\epsilon}_{[i]}^{\text {upp }}$ and $\bar{\epsilon}_{[i]}^{\text {low }} \in \mathbb{R}_{+}^{n_{i}}$. If the residual lies in the interval between the thresholds, then the subsystem is said to be "healthy" with a certain probability; otherwise, if it crosses one of the two thresholds, we say that a fault has probably occurred. The diagnosers are designed so to guarantee the convergence of the mean of the estimation error.

\section{A. The Fault Detection Estimator}

For fault detection purposes, each subsystem is monitored by a local nonlinear estimator, based on the local model $\Sigma_{[i]}$ in (1). The $k_{i}$-th non-shared state variable of $\Sigma_{[i]}$ can be estimated as

$$
\hat{x}_{\left[i, k_{i}\right]}^{+}=\lambda\left(\hat{x}_{\left[i, k_{i}\right]}-y_{\left[i, k_{i}\right]}\right)+f_{i, k_{i}}\left(y_{[i]}, z_{[i]}, u_{[i]}\right),
$$

where the filter parameter is chosen in the interval $0<\lambda<$ 1 , in order to guarantee convergence properties. Let now consider a shared variable $x_{\left[i, k_{i}\right]}=x_{\left[j, k_{j}\right]}$, where $k_{i}$ and $k_{j}$ are the $k_{i}$-th and $k_{j}$-th components of local vectors $x_{[i]}$ and $x_{[j]}$, respectively. We use the redundant measurements due to overlapping for implementing a deterministic consensus approach (see [12] where the effectiveness of this consensus approach is demonstrated for a stochastic framework). In fact, as regards shared variables estimation, each subsystem communicates with parents and children subsystems sharing that variable. In the following, $\mathbb{S}^{k}$ is the set of subsystems $\Sigma_{[i]}$ sharing a given state variable $k$ of the LSS. Let the shared variable be $x_{\left[i, k_{i}\right]}$. The estimates of shared variables are provided by the following estimation model:

$\hat{x}_{\left[i, k_{i}\right]}^{+}=\sum_{j \in \mathbb{S}^{k}} W_{i, j}^{k}\left[\lambda\left(\hat{x}_{\left[j, k_{j}\right]}-y_{\left[j, k_{j}\right]}\right)+f_{j, k_{j}}\left(y_{[j]}, z_{[j]}, u_{[j]}\right)\right]$

where $W_{i, j}^{k}$ are the components of a time-varying rowstochastic matrix $W^{k}$, which will be defined in Subsection III-C, designed to reduce variance uncertainty of the FD thresholds ${ }^{1}$.

\section{B. The detection thresholds}

In order to properly define the probabilistic upper and lower thresholds for FD, we analyze the dynamics of the local diagnoser estimation error in healthy conditions. Defining $W^{k}$ such that $\sum_{j \in \mathbb{S}^{k}} W_{i, j}^{k}=1$ and since for shared variables $\forall i, j \in \mathbb{S}^{k}$ there are $k_{i}$ and $k_{j}$ such that it holds $f_{i, k_{i}}\left(x_{[i]}, \psi_{[i]}, u_{[i]}\right)=f_{j, k_{j}}\left(x_{[j]}, \psi_{[j]}, u_{[j]}\right)$, the $k$-th state estimation error dynamics model is given $b^{2}$

\footnotetext{
${ }^{1}$ We note that (3) is a general representation that holds also for the case of non-shared variables (2), when $\mathbb{S}^{k}=\{i\}$, and $W_{i, i}^{k}=1$ by definition.

${ }^{2}$ In the following, for the sake of simplicity, we omit the subscript of the shared component index $k$, i.e. we use $x_{[i, k]}$ instead of $x_{\left[i, k_{i}\right]}$ when it is not strictly necessary.
} 


$$
\epsilon_{[i, k]}^{+}=\sum_{j \in \mathbb{S}^{k}} W_{i, j}^{k}\left[\lambda \epsilon_{[j, k]}+\Delta f_{j, k}+w_{j, k}+\varrho_{[i, k]}^{+}\right],
$$

where $\Delta f_{j, k} \triangleq f_{j, k}\left(x_{[j]}, \psi_{[j]}, u_{[j]}\right)-f_{j, k}\left(y_{[j]}, z_{[j]}, u_{[j]}\right)$ and $\varrho_{[i, k]}^{+}$is the measurement error at time $t+1$. This is the general formulation, holding also for non-shared variables:

$$
\epsilon_{[i, k]}^{+}=\lambda \epsilon_{[i, k]}+\Delta f_{i, k}+w_{i, k}+\varrho_{[i, k]}^{+} .
$$

We now analyze the stochastic part of the residual:

$$
\chi_{[i, k]}^{+}=\Delta f_{i, k}+w_{i, k}+\varrho_{[i, k]}^{+} .
$$

Its mean and variance can be computed as

$$
\begin{gathered}
\mathbb{E}\left[\chi_{[i, k]}^{+}\right]=\mathbb{E}\left[\Delta f_{i, k}\right]+\mathbb{E}\left[w_{i, k}\right]+\mathbb{E}\left[\varrho_{[i, k]}^{+}\right] \\
\operatorname{Var}\left[\chi_{[i, k]}^{+}\right]=\operatorname{Var}\left[\Delta f_{i, k}\right]+\operatorname{Var}\left[w_{i, k}\right]+\operatorname{Var}\left[\varrho_{[i, k]}^{+}\right] \\
+2 \operatorname{Cov}\left[\Delta f_{i, k}, \varrho_{[i, k]}^{+}\right]
\end{gathered}
$$

The following further assumptions are needed.

Assumption 3: The measurement noise $\varrho_{[i, k]}$ and the modeling uncertainty $w_{i, k}$ are not correlated.

Assumption 4: Given the values of $y_{[i]}, z_{[i]}, u_{[i]}$ and known the probabilistic distribution of $\varrho_{[i]}$ (and so of $\theta_{[i]}$ ), it is possible to compute $\mathbb{E}\left[\Delta f_{i, k}\right]$ and $\operatorname{Var}\left[\Delta f_{i, k}\right]$.

In the linear case, the solution of this problem is trivial and it is not necessary to know the measurement noise distribution. For the sake of simplicity, we consider the following non restrictive assumption, permitting to simplify Eq.(6).

Assumption 5: The measurement noise and the modeling uncertainty are zero-mean: $\mu_{\varrho_{[i]}}(t)=0, \mu_{w_{i}}(t)=0, \forall t$.

We now derive suitable time-varying probabilistic bounds for $\chi_{[i, k]}^{+}$using Chebyshev inequalities, without requiring any assumption on the distribution of the residual. Better results could be found in case of known distribution of the residual process. For a stochastic variable $X$, with mean $\mu(X)$ and standard deviation $\sigma(X)$, it holds:

$$
\operatorname{Pr}(\mu(X)-\alpha \sigma(X) \leq X \leq \mu(X)+\alpha \sigma(X)) \geq 1-1 / \alpha^{2}
$$

where $\alpha>1$ is a tunable, real scalar. Therefore, it is possible to obtain a lower and a upper stochastic thresholds for the residual signal, so that at each time $t$

$$
\bar{\epsilon}_{[i]}^{\text {low }} \leq \epsilon_{[i]} \leq \bar{\epsilon}_{[i]}^{\text {upp }} \text {. }
$$

The thresholds can be computed, in the general shared case, at each step $t$ for the following step $t+1$ as:

$$
\begin{array}{r}
\bar{\epsilon}_{[i, k]}^{+ \text {upp } / \text { low }}=\sum_{j \in \mathbb{S}^{k}} W_{i, j}^{k}\left[\lambda \bar{\epsilon}_{[j, k]}^{\text {upp low }}+\mathbb{E}\left[\Delta f_{j, k}\right]\right] \\
\pm \alpha\left\{\sum _ { j \in \mathbb { S } ^ { k } } ( W _ { i , j } ^ { k } ) ^ { 2 } \left[\operatorname{Var}\left[\Delta f_{j, k}\right]+\sigma_{w_{j, k}}^{2}+\sigma_{\varrho_{[j, k]}^{+}}^{2}\right.\right. \\
\left.\left.+2 \operatorname{Cov}\left[\Delta f_{j, k}, \varrho_{[j, k]}^{+}\right]\right]\right\}^{\frac{1}{2}} .
\end{array}
$$

The value of $\alpha$ is a tuning parameter by which different values of guaranteed false-alarms rate can be set.

\section{The consensus matrix}

In this subsection, we explain how to design the timevarying consensus matrix.

Each row and each column represent a diagnoser sharing the variable $k$ : the generic element $W_{i, j}^{k}$ indicates how much the $i$-th diagnoser weights the consensus terms received by the $j$-th diagnoser in $\mathbb{S}^{k}$. We define the time-varying consensus-weighting matrix $W^{k}$ for each $(i, j)$-th component. The objective is to obtain the most reliable local state estimation by using the terms communicated by subsystems in $\mathbb{S}^{k}$ at the current time step. To do that, we want to find the weights that allow to minimize the thresholds (9), by weighting more the subsystems that have lower uncertainty in its measurements and in the local model. The consensus matrix can be time-varying due to the changes on the uncertainty level. Since the amplitude of the thresholds is mainly due to the variance terms in (9), we decide to minimize those terms. This is obtained by solving the following quadratic optimization problem:

$$
\begin{aligned}
\min _{W_{i, j}^{k}} & \sum_{j \in \mathbb{S}^{k}}\left(W_{i, j}^{k}\right)^{2} \operatorname{Var}\left[\chi_{[j, k]}\right] \\
\text { s. t. } & \sum_{j \in \mathbb{S}^{k}} W_{i, j}^{k}=1, \\
& \left|W_{i, j}^{k}\right| \leq 1 \quad \forall j \in \mathbb{S}^{k} .
\end{aligned}
$$

We have the following result. The proof is omitted due to length constraints.

Proposition 1: The optimal weights for the minimization problem in (10) are, $\forall j \in \mathbb{S}^{k}$ :

$$
W_{i, j}^{k}=\frac{1}{\operatorname{Var}\left[\chi_{[j, k]}\right]\left(\sum_{j} \frac{1}{\operatorname{Var}\left[\chi_{[j, k]}\right]}\right)} .
$$

At each time-step, every local fault-diagnoser receives estimates and consensus terms of variable $x_{[i, k]}$ from the subsystems sharing it. Then, it selects and weights the contributions affected by "smaller uncertainty".

\section{Estimator convergence}

Next, we address the convergence properties of the overall estimator before the possible occurrence of a fault, that is for $t<T_{0}$. Towards this end, for analysis purposes, we introduce the extended estimation error vector $\epsilon_{k, E}$, which is a column vector collecting the estimation error vectors of the $N_{k}$ subsystems sharing the $k$-th state component: $\epsilon_{k, E} \triangleq$ $\operatorname{col}\left(\epsilon_{[j, k]}: j \in \mathbb{S}^{k}\right)$. Hence, the dynamics of $\epsilon_{k, E}$ can be described as:

$$
\epsilon_{k, E}^{+}=W^{k}\left[\lambda \epsilon_{k, E}+\Delta f_{k, E}+w_{k, E}\right]+\varrho_{k, E}^{+},
$$

where $\varrho_{k, E}$ is a column vector, collecting the corresponding $k_{j}$ value of vector $\varrho_{[j]}$, i.e. $\varrho_{\left[j, k_{J}\right]}$, for each $j \in \mathbb{S}^{k} ; \Delta f_{k, E}$ and $w_{k, E}$ are column vectors collecting the vectors $w_{j, k}$ and $\Delta f_{j, k}$, with $j \in \mathbb{S}^{k}$, respectively. The following convergence result can now be provided. The proof is omitted due to space constraints.

Proposition 2: System (12), describing the mean of the estimation error dynamics, being the consensus matrix rowstochastic and $0<\lambda<1$, is Bounded Input Bounded Output. 


\section{E. Fault Detectability analysis}

In this section we derive some detectability conditions. In this case, the residual in (5) can be written as:

$$
\begin{array}{r}
\epsilon_{[i, k]}(t)=\sum_{h=0}^{t-1} \lambda^{t-1-h}\left(\Delta f_{i, k}(h)+w_{i, k}(h)+\varrho_{[i, k]}(h+1)\right. \\
\left.+\phi_{i, k}(h)\right)+\lambda^{t} \epsilon_{[i, k]}(0)
\end{array}
$$

As $\phi_{i, k}(t)=\phi_{i, k}\left(x_{[i]}, \psi_{[i]}, u_{[i]}, t\right)=0$ (with some abuse of notation) for $t<T_{0}$, the residual can be rewritten as

$$
\epsilon_{[i, k]}(t)=U_{i, k}(t)+\sum_{h=T_{0}}^{t-1} \lambda^{t-1-h} \phi_{i, k}(h)
$$

where $U_{i, k}(t)$ represents the part of the residual collecting all the uncertainty terms, not including fault dynamics:

$$
\begin{array}{r}
U_{i, k}(t)=\sum_{h=0}^{t-1} \lambda^{t-1-h}\left(\chi_{[i, k]}(h+1)\right)+\lambda^{t} \epsilon_{[i, k]}(0) \\
=\sum_{h=0}^{t-1} \lambda^{t-1-h}\left(\chi_{[i, k]}(h+1)\right),
\end{array}
$$

since $\hat{x}_{[i, k]}(0)=y_{[i]}(0)$ and then $\epsilon_{[i, k]}(0)=0$. The threshold, as by definition in (8), is designed so that

$$
\bar{\epsilon}_{[i, k]}^{\text {low }}(t) \leq U_{i, k}(t) \leq \bar{\epsilon}_{[i, k]}^{\text {upp }}(t)
$$

with a certain probability depending on $\alpha$. A fault is detected at a certain time instant $t=T_{d}>T_{0}$ (detection time) if

$$
\epsilon_{[i, k]}(t) \notin\left(\bar{\epsilon}_{[i, k]}^{\text {low }}(t), \bar{\epsilon}_{[i, k]}^{\text {upp }}(t)\right)
$$

for at least one state component $k \in\left\{1: n_{i}\right\}$. Following (14), condition (15) is equivalent to:

$$
\begin{aligned}
\sum_{h=T_{0}}^{t-1} \lambda^{t-1-h} & \phi_{i, k}(h) \\
& \notin\left(\bar{\epsilon}_{[i, k]}^{l o w}(t)-U_{i, k}(t), \bar{\epsilon}_{[i, k]}^{u p p}(t)-U_{i, k}(t)\right) .
\end{aligned}
$$

The uncertainty term can be expressed as

$$
\chi_{[i, k]}(t)=\mathbb{E}\left[\chi_{[i, k]}(t)\right]+\Delta \chi_{[i, k]}(t),
$$

where $\Delta \chi_{[i, k]}$ is the deviation of the uncertainty from its mean, and the thresholds defined in (9) can be rewritten as

$$
\begin{gathered}
\bar{\epsilon}_{[i, k]}^{\text {upp } / \text { low }}(t)=\sum_{h=0}^{t-1} \lambda^{t-1-h}\left(\mathbb{E}\left[\chi_{[i, k]}(h+1)\right]\right. \\
\left. \pm \alpha\left[\operatorname{Var}\left[\chi_{[i, k]}(h+1)\right]\right]^{\frac{1}{2}}\right)+\lambda^{t} \bar{\epsilon}_{[i, k]}^{\text {upp } / \text { low }}(0),
\end{gathered}
$$

where the thresholds are initialized with $\bar{\epsilon}_{[i, k]}^{\text {upp/low }}(0)=0$. The detectability conditions become:

$$
\begin{aligned}
& \sum_{h=T_{0}}^{t-1} \lambda^{t-1-h} \phi_{i, k}(h) \notin \sum_{h=T_{0}}^{t-1} \lambda^{t-1-h}\left(-\alpha\left[\operatorname{Var}\left[\chi_{[i, k]}(h+1)\right]\right]^{\frac{1}{2}}\right. \\
& \left.-\Delta \chi_{[i, k]}(h+1),+\alpha\left[\operatorname{Var}\left[\chi_{[i, k]}(h+1)\right]\right]^{\frac{1}{2}}-\Delta \chi_{[i, k]}(h+1)\right) .
\end{aligned}
$$

Since $\Delta \chi_{[i, k]}(t)$ is zero-mean, using Chebishev inequalities we obtain

$$
-\alpha\left[\operatorname{Var}\left[\chi_{[i, k]}(t)\right]\right]^{\frac{1}{2}} \leq \Delta \chi_{[i, k]}(t) \leq \alpha\left[\operatorname{Var}\left[\chi_{[i, k]}(t)\right]\right]^{\frac{1}{2}}
$$

with a certain probability depending on $\alpha$. Therefore, the fault detection is guaranteed at time $T_{d}$ with a certain falsealarms rate depending on $\alpha$, when the following detectability condition is satisfied:

$$
\begin{aligned}
& \left|\sum_{h=T_{0}}^{T_{d}-1} \lambda^{T_{d}-1-h} \phi_{i, k}(h)\right|> \\
& 2 \alpha \sum_{h=T_{0}}^{T_{d}-1} \lambda^{T_{d}-1-h}\left[\operatorname{Var}\left[\chi_{[i, k]}(h+1)\right]\right]^{\frac{1}{2}} .
\end{aligned}
$$

In this way, we have derived a characterization in a nonclosed form of a class of faults that can be detected given some uncertainty conditions.

We can then obtain a detection condition in closed form. We have the following result. Due to space constraints the proof is omitted.

Proposition 3: Let us assume that a fault $\phi_{i, k}\left(x_{[i]}, \psi_{[i]}, u_{[i]}, t\right)$ is occurring on the $k$-th variable of the $i$-th subsystem. The fault will be detected at a certain time $T_{e}$ if

$$
\begin{gathered}
\phi_{i, k}\left(x_{[i]}, \psi_{[i]}, u_{[i]}, T_{e}-1\right) \notin\left(\lambda r_{[i, k]}^{l o w}\left(T_{e}-1\right)\right. \\
\left.-2 \alpha \operatorname{Var}\left[\chi_{[i, k]}\left(T_{e}\right)\right]^{\frac{1}{2}}, \lambda r_{[i, k]}^{u p p}\left(T_{e}-1\right)+2 \alpha \operatorname{Var}\left[\chi_{[i, k]}\left(T_{e}\right)\right]^{\frac{1}{2}}\right)
\end{gathered}
$$

where $r_{[i, k]}^{\text {upp/low }}:=\bar{\epsilon}_{[i, k]}^{\text {upp /low }}-\epsilon_{[i, k]}$ is the distance of the residual from the threshold.

\section{Distributed Fault Isolation}

We now propose a novel distributed Fault Isolation scheme in a stochastic uncertainty framework. The fault isolation architecture consists in kind of a Generalized Observer Scheme (GOS, see [18]). Similarly as in [8], we assume that each subsystem knows a local fault set $\mathcal{F}_{i}$, collecting all the $N_{\mathcal{F}_{i}}$ possible nonlinear fault functions: $\phi_{i}^{l}\left(x_{[i]}, \psi_{[i]}, u_{[i]}, t\right)$, $l \in\left\{1, \ldots, N_{\mathcal{F}_{i}}\right\}$. After fault detection, each interested LFD uses $N_{\mathcal{F}_{i}}$ nonlinear estimators of the local state $x_{[i]}$ in order to locally determine which of the possible $N_{\mathcal{F}_{i}}$ faults in the set $\mathcal{F}_{i}$ has occurred.

Differently from previous works (see [8], [12]), here the uncertainties are not bounded by deterministic bounds, but instead are modeled as stochastic processes. Therefore, we design some probabilistic thresholds for the distributed fault isolation task and we analyze the probability that a certain fault has occurred in the considered subsystem. In this paper, we consider local faults, but also the distributed ones can be managed with the same approach, each local diagnoser trying to isolate the local part of the distributed fault.

Specifically, once a fault is detected at time $T_{d}$ in the $i$-th subsystem, each involved diagnoser activates its $N_{\mathcal{F}_{i}}$ estimators, where each filter is sensitive to a specific fault. Each $l$-th estimator provides a local state estimate $\hat{x}_{[i]}^{l}$ of the local state $x_{[i]}$ affected by the $l$-th fault. The difference between the estimate $\hat{x}_{[i]}^{l}$ and the measurements $y_{[i]}$ consist 
in the fault isolation estimation error $\epsilon_{[i]}^{l} \triangleq y_{[i]}-\hat{x}_{[i]}^{l}$, used as a residual and compared, component by component, to some properly designed probabilistic isolation thresholds $\bar{\epsilon}_{[i]}^{l}$ upp/low $\in \mathbb{R}_{+}^{n_{i}}$. We derive a lower and a upper stochastic thresholds, so that at each time $t$, with a certain probability

$$
\bar{\epsilon}_{[i]}^{l \text { low }} \leq \epsilon_{[i]}^{l} \leq \bar{\epsilon}_{[i]}^{l \text { upp }} .
$$

The thresholds can be computed at each step $t$ for the following step $t+1$. If the residual crosses one of the two thresholds, that is

$$
\epsilon_{[i, k]}^{l}(t) \notin\left(\bar{\epsilon}_{[i, k]}^{l \text { low }}(t), \bar{\epsilon}_{[i, k]}^{l \text { upp }}(t)\right)
$$

we can exclude the occurrence of the considered $l$-th fault, with a certain guaranteed probability error (see Section IV-C for the complete analysis). If we are able to exclude all the faults but one, then we can say that the fault is isolated with a certain probability.

\section{A. The Fault Isolation Estimators}

After the fault $\phi_{i}$ has occurred, the dynamics of the $k$-th state component of the $i$-th subsystem becomes

$$
x_{[i, k]}^{+}=f_{i, k}\left(x_{[i]}, \psi_{[i]}, u_{[i]}\right)+w_{i, k}+\phi_{i, k}\left(x_{[i]}, \psi_{[i]}, u_{[i]}, t\right),
$$

being $\phi_{i, k} \neq 0$. The $l$-th estimate for the general case of a fault on a shared variable is

$$
\begin{array}{r}
\hat{x}_{[i, k]}^{+l}=\sum_{j \in \mathbb{S}^{k}} W_{i, j}^{l k}\left[\lambda\left(\hat{x}_{[j, k]}^{l}-y_{[j, k]}^{l}\right)+f_{j, k}\left(y_{[j]}, z_{[j]}, u_{[j]}\right)\right. \\
\left.+\phi_{j, k}^{l}\left(y_{[j]}, z_{[j]}, u_{[j]}, t\right)\right] .
\end{array}
$$

The corresponding estimation error dynamic equation is

$\epsilon_{[i, k]}^{l+}=\sum_{j \in \mathbb{S}^{k}} W_{i, j}^{l k}\left[\lambda \epsilon_{[j, k]}^{l}+\Delta f_{j, k}+w_{j, k}+\Delta \phi_{j, k}^{l}+\varrho_{[j, k]}^{+}\right]$,

being

$$
\Delta \phi_{j, k}^{l}=\phi_{j, k}\left(x_{[j]}, \psi_{[j]}, u_{[j]}, t\right)-\phi_{j, k}^{l}\left(y_{[j]}, z_{[j]}, u_{[j]}, t\right) .
$$

\section{B. Fault Isolation thresholds}

In the matched case, that is, $\phi_{i, k}=\phi_{i, k}^{l}\left(x_{[i]}, \psi_{[i]}, u_{[i]}, t\right)$, we can use a similar logic as in Section III-B and define an upper and a lower isolation thresholds for each $l$-th residual signal, basing on the Chebyshev law:

$$
\begin{gathered}
\bar{\epsilon}_{[i, k]}^{l+\text { upp } / \text { low }}=\lambda \bar{\epsilon}_{[i, k]}^{l \text { upp } / \text { low }}+\mathbb{E}\left[\chi_{[i, k]}^{\phi^{l}+}\right] \pm \alpha\left[\operatorname{Var}\left[\chi_{[i, k]}^{\phi^{l}+}\right]\right]^{\frac{1}{2}} \\
=\lambda \bar{\epsilon}_{[i, k]}^{l \text { upp } / \text { low }}+\mathbb{E}\left[\Delta f_{i, k}+\Delta \phi_{i, k}^{l}\right] \pm \alpha\left[\operatorname{Var}\left[\Delta f_{i, k}\right]\right. \\
+\operatorname{Var}\left[\Delta \phi_{i, k}^{l}\right]+\sigma_{w_{i, k}}^{2}+\sigma_{\varrho_{[i, k]}^{+}}^{2}+2 \operatorname{Cov}\left[\Delta f_{i, k}, \varrho_{[i, k]}^{+}\right] \\
\left.+2 \operatorname{Cov}\left[\Delta \phi_{i, k}^{l}, \varrho_{[i, k]}^{+}\right]+2 \operatorname{Cov}\left[\Delta f_{i, k}, \Delta \phi_{i, k}^{l}\right]\right]^{\frac{1}{2}}
\end{gathered}
$$

where $\chi_{[i, k]}^{\phi^{l}+}=\Delta f_{i, k}+w_{i, k}+\Delta \phi_{i, k}^{l}+\varrho_{[i, k]}^{+}$.

Assumption 6: Given the values of $y_{[i]}, z_{[i]}, u_{[i]}$ and known the probabilistic distribution of $\varrho_{[i]}$ (and so of $\left.\theta_{[i]}\right)$, it is possible to compute $\mathbb{E}^{3}\left[\Delta \phi_{i, k}^{l}\right], \operatorname{Var}\left[\Delta \phi_{i, k}^{l}\right]$,

\footnotetext{
${ }^{3}$ For example, Monte Carlo methods, propagation of error formula or the unscented transform can be used. Same remark holds for Assumption 4.
}

$\operatorname{Cov}\left[\Delta \phi_{i, k}^{l}, \varrho_{[i, k]}^{+}\right]$and $\operatorname{Cov}\left[\Delta f_{i, k}, \Delta \phi_{i, k}^{l}\right]$, where $\Delta \phi_{i, k}^{l}=$ $\phi_{i, k}^{l}\left(y_{[i]}-\varrho_{[i]}, z_{[i]}-\theta_{[i]}, u_{[i]}\right)-\phi_{i, k}^{l}\left(y_{[i]}, z_{[i]}, u_{[i]}\right)$ is stochastic because of the measurement error $\varrho_{[i]}$.

In the shared variables case we have

$$
\begin{aligned}
\bar{\epsilon}_{[i, k]}^{l+u p p / l o w}= & \sum_{j \in \mathbb{S}^{k}} W_{i, j}^{l k}\left[\lambda \bar{\epsilon}_{[i, k]}^{l \text { upp } / \text { low }}+\mathbb{E}\left[\chi_{[i, k]}^{\phi^{l}+}\right]\right] \\
& \pm \alpha\left\{\sum_{j \in \mathbb{S}^{k}}\left(W_{i, j}^{l k}\right)^{2}\left[\operatorname{Var}\left[\chi_{[i, k]}^{\phi^{l}+}\right]\right]\right\}^{\frac{1}{2}}
\end{aligned}
$$

As for the detection case (see (10) in Section III-C), here for fault isolation estimators we propose to define the time varying consensus matrix elements $W_{i, j}^{l k}$, for each $l$-th isolation estimator, so to minimize the variance terms of the isolation thresholds:

$$
W_{i, j}^{l k}=\frac{1}{\operatorname{Var}\left[\chi_{[j, k]}^{\phi^{l}}\right]\left(\sum_{j} \frac{1}{\operatorname{Var}\left[\chi_{[j, k]}^{\phi^{l}}\right]}\right)}, \quad \forall j \in \mathbb{S}^{k} .
$$

\section{Fault exclusion error probability analysis}

By the definition of the thresholds in (19), the probability that the residual $\epsilon_{[i, k]}^{l}$ lies inside the thresholds interval at a certain time $t$, assuming that the fault is matched, that is $\phi_{i, k}=\phi_{i, k}^{l}\left(x_{[i]}, \psi_{[i]}, u_{[i]}, t\right)$, is

$\operatorname{Pr}\left(\epsilon_{[i, k]}^{l}(t) \in\left(\bar{\epsilon}_{[i, k]}^{l \text { low }}(t), \bar{\epsilon}_{[i, k]}^{l}\right.\right.$ upp $\left.\left.(t)\right) \mid \phi_{i, k}=\phi_{i, k}^{l}\right) \geq 1-\frac{1}{\alpha^{2}}$.

Therefore, the false alarm probability can be computed as follows. The probability that the residual $\epsilon_{[i, k]}^{l}$ crosses one of the related thresholds $\bar{\epsilon}_{[i, k]}^{l+\text { upp/low }}$, thus excluding the $l$-th fault in the case that the fault is matched, is lower than $\frac{1}{\alpha^{2}}$ :

$$
\operatorname{Pr}\left(\epsilon_{[i, k]}^{l}(t) \notin\left(\bar{\epsilon}_{[i, k]}^{l \text { low }}(t), \bar{\epsilon}_{[i, k]}^{l} \text { upp }(t)\right) \mid \phi_{i, k}=\phi_{i, k}^{l}\right) \leq \frac{1}{\alpha^{2}} .
$$

This is the probability of mistakenly excluding the $l$-th fault using thresholds $\bar{\epsilon}_{[i, k]}^{l+u p p / l o w}$. We can therefore set $\alpha$ depending of the maximum error probability we can accept.

Finally, the proposed monitoring architecture can show better performance if we assume to know for each $l$-th fault the probability that it occurs at a certain time $t: \operatorname{Pr}\left(\phi_{i, k}=\right.$ $\left.\phi_{i, k}^{l}\right)$. The probability to have a correct fault isolation is therefore

$$
\begin{array}{r}
\operatorname{Pr}\left(\epsilon_{[i, k]}^{l}(t) \in\left(\bar{\epsilon}_{[i, k]}^{l \text { low }}(t), \bar{\epsilon}_{[i, k]}^{l \text { upp }}(t)\right) \cap \phi_{i, k}=\phi_{i, k}^{l}\right) \\
=\operatorname{Pr}\left(\epsilon_{[i, k]}^{l}(t) \in\left(\bar{\epsilon}_{[i, k]}^{l \text { low }}(t), \bar{\epsilon}_{[i, k]}^{l \text { upp }}(t)\right) \mid \phi_{i, k}=\phi_{i, k}^{l}\right) \\
\quad \times \operatorname{Pr}\left(\phi_{i, k}=\phi_{i, k}^{l}\right) \geq\left(1-\frac{1}{\alpha^{2}}\right) \operatorname{Pr}\left(\phi_{i, k}=\phi_{i, k}^{l}\right),
\end{array}
$$

thanks to the theorem of compound probability.

Furthermore, it is worth noting that the distance of the residual from the thresholds gives us some useful information. Given the mean and the variance of the theoretical residual at a given time $t$, we can compute the probability that the current measurement is explained by the considered 
model. Computing

$$
\alpha_{l}=\frac{\epsilon_{[i, k]}^{l}-\left(\lambda \bar{\epsilon}_{[i, k]}^{l \text { upp } / \text { low }}+\mathbb{E}\left[\chi_{[i, k]}^{\phi^{l}+}\right]\right)}{\left(\operatorname{Var}\left[\chi_{[i, k]}^{\phi^{l}+}\right]\right)^{\frac{1}{2}}},
$$

if $\alpha_{l}>1$, it follows that

$$
\begin{aligned}
\operatorname{Pr}\left(\epsilon_{[i, k]}^{l}(t) \notin\left(\bar{\epsilon}_{[i, k]}^{l \text { low }}\left(\alpha_{l}, t\right), \bar{\epsilon}_{[i, k]}^{l \text { upp }}\left(\alpha_{l}, t\right)\right)\right. & \left.\mid \phi_{i, k}=\phi_{i, k}^{l}\right) \\
\leq \frac{1}{\alpha_{l}^{2}} . &
\end{aligned}
$$

We can in this way define some new thresholds using $\alpha_{l}$ and compute the error probability that we get by excluding or accepting the $l$-th fault.

\section{Fault isolability}

Let us now consider the case of a non-matched fault, that is, $\phi_{i, k}=\phi_{i, k}^{p}\left(x_{[i]}, \psi_{[i]}, u_{[i]}, t\right)$, with $p \neq l$. Then, in the case of a non-shared variable $k$, the dynamics of the estimation error of the $l$-th fault isolation estimator for each $i$-th subsystem can be modelled as

$$
\epsilon_{[i, k]}^{l+}=\lambda \epsilon_{[i, k]}^{l}+\Delta f_{i, k}+w_{i, k}+\Delta \phi_{i, k}^{p / l}+\varrho_{[i, k]}^{+},
$$

where

$$
\Delta \phi_{i, k}^{p / l}=\phi_{i, k}^{p}\left(x_{[i]}, \psi_{[i]}, u_{[i]}, t\right)-\phi_{i, k}^{l}\left(y_{[i]}, z_{[i]}, u_{[i]}, t\right) .
$$

Instead, for the shared case we have

$$
\epsilon_{[i, k]}^{l+}=\sum_{j \in \mathbb{S}^{k}} W_{i, j}^{l k}\left[\lambda \epsilon_{[j, k]}^{l}+\Delta f_{j, k}+w_{j, k}+\Delta \phi_{j, k}^{p / l}+\varrho_{[j, k]}^{+}\right] .
$$

For the sake of notational simplicity, we now continue the analysis only for non-shared variables. It is anyway simple to extend in the general case of shared state components. We have the following result:

Proposition 4: Given a fault $\phi_{i, k}^{p}\left(x_{[i]}, \psi_{[i]}, u_{[i]}, t\right)$ occurring on the $k$-th variable of the $i$-th subsystem, the $l$-th fault isolation estimator will exclude the $l$-th fault function, with $l \in N_{\mathcal{F}_{i}}$, if at a certain time $T_{e}$

$$
\begin{gathered}
\phi_{i, k}^{p}\left(x_{[i]}, \psi_{[i]}, u_{[i]}, T_{e}-1\right) \notin\left(\lambda r_{[i, k]}^{l}\left(T_{e}-1\right)+\mathbb{E}\left[\Delta \phi_{i, k}^{l}\right]\right. \\
-\alpha\left(\operatorname{Var}\left[\chi_{[i, k]}^{\phi^{l}}\left(T_{e}\right)\right]^{\frac{1}{2}}+\operatorname{Var}\left[\chi_{[i, k]}\left(T_{e}\right)\right]^{\frac{1}{2}}\right) \\
+\phi_{i, k}^{l}\left(y_{[i]}, z_{[i]}, u_{[i]}, T_{e}-1\right), \lambda r_{[i, k]}^{l}\left(T_{e}-1\right)+\mathbb{E}\left[\Delta \phi_{i, k}^{l}\right] \\
+\alpha\left(\operatorname{Var}\left[\chi_{[i, k]}^{\phi^{l}}\left(T_{e}\right)\right]^{\frac{1}{2}}+\operatorname{Var}\left[\chi_{[i, k]}\left(T_{e}\right)\right]^{\frac{1}{2}}\right) \\
\left.+\phi_{i, k}^{l}\left(y_{[i]}, z_{[i]}, u_{[i]}, T_{e}-1\right)\right)
\end{gathered}
$$

where $r_{[i, k]}^{l}:=\bar{\epsilon}_{[i, k]}^{l+\text { upp/low }}-\epsilon_{[i, k]}^{l+}$ is the distance of the residual from the threshold at the previous step $T_{e}-1$.

\section{CONCLUding REMARKS}

In this paper, a stochastic distributed fault detection and isolation architecture for nonlinear LSSs is designed. The proposed monitoring architecture is based on the model of the large-scale system, composed of several interconnected subsystems. The modeling uncertainties and measurement noises are represented as stochastic processes. Each subsystem is monitored by a local diagnoser. Fault detection and isolation probabilistic thresholds are designed, guaranteeing error levels set by the designer. Fault detectability and isolability analysis are provided.

Future research efforts will be devoted to test the effectiveness of the proposed FDI approach in simulation. Early simulation results have been obtained but they could not be included in the present paper due to length limitations.

\section{REFERENCES}

[1] J. Lunze, Feedback control of large scale systems. Upper Saddle River, NJ, USA: Prentice Hall, Systems and Control Engineering, 1992.

[2] T. Samad and T. Parisini, "Systems of Systems," in The Impact of Control Technology, T. Samad and A. M. Annaswamy, Eds. IEEE Control Systems Society, 2011, pp. 175-183. [Online]. Available: ieeecss.org/general/impact-control-technology

[3] K. Baheti and H. Gill, "Cyber-physical Systems," in The Impact of Control Technology, T. Samad and A. M. Annaswamy, Eds. IEEE Control Systems Society, 2011, pp. 161-166. [Online]. Available: http://ieeecss.org/general/impact-control-technology

[4] R. J. Patton, C. Kambhampati, A. Casavola, P. Zhang, S. Ding, and D. Sauter, "A generic strategy for fault-tolerance in control systems distributed over a network," European Journal of Control, vol. 13, no. 2-3, pp. 280-296, 2007.

[5] W. Li, W. Gui, Y. Xie, and S. Ding, "Decentralized fault detection system design for large-scale interconnected systems," in Proc. of the 7th IFAC Symposium on Fault Detection, Supervision and Safety of Technical Processes, 2009, pp. 816-821.

[6] X. Zhang and Q. Zhang, "Distributed fault diagnosis in a class of interconnected nonlinear uncertain systems," International Journal of Control, vol. 85, no. 11, pp. 1644-1662, 2012.

[7] F. Boem, R. M. G. Ferrari, and T. Parisini, "Distributed fault detection and isolation of continuous-time nonlinear systems," Europ. J. of Control, no. 5-6, pp. 603-620, 2011.

[8] R. M. G. Ferrari, T. Parisini, and M. M. Polycarpou, "Distributed Fault Detection and Isolation of Large-Scale Discrete-Time Nonlinear Systems: An Adaptive Approximation Approach," IEEE Transactions on Automatic Control, vol. 57, no. 2, pp. 275-290, 2012.

[9] V. Reppa, M. Polycarpou, and C. Panayiotou, "Distributed sensor fault diagnosis for a network of interconnected cyberphysical systems," Control of Network Systems, IEEE Transactions on, vol. 2, no. 1, pp. 11-23, March 2015.

[10] M. Staroswiecki and A. M. Amani, "Fault-tolerant control of distributed systems by information pattern reconfiguration," International Journal of Adaptive Control and Signal Processing, 2014.

[11] C. Keliris, M. M. Polycarpou, and T. Parisini, "Distributed fault diagnosis for process and sensor faults in a class of interconnected input-output nonlinear discrete-time systems," International Journal of Control, no. just-accepted, pp. 1-26, 2015.

[12] F. Boem, S. Riverso, G. Ferrari-Trecate, and T. Parisini, "A Plug-andPlay Fault Diagnosis Approach for Large-Scale Systems," in IFAC Safeprocess Conf., 2015.

[13] S. Yin, S. X. Ding, X. Xie, and H. Luo, "A review on basic data-driven approaches for industrial process monitoring," Industrial Electronics, IEEE Transactions on, vol. 61, no. 11, pp. 6418-6428, 2014.

[14] P. M. Frank, "Analytical and qualitative model-based fault diagnosis-a survey and some new results," European Journal of control, vol. 2, no. 1, pp. 6-28, 1996.

[15] S. Yin, X. Yang, and H. R. Karimi, "Data-driven adaptive observer for fault diagnosis," Mathematical Problems in Engineering, vol. 2012, 2012.

[16] F. Boem, R. Ferrari, T. Parisini, and M. Polycarpou, "Optimal Topology for Distributed Fault Detection of Large-scale Systems," in IFAC Safeprocess Conf., 2015.

[17] F. Boem, S. Riverso, G. Ferrari-Trecate, and T. Parisini, "Stochastic fault detection in a plug and play scenario," in Conf. on Decision and Control, (accepted), 2015.

[18] R. Patton, P. Frank, and D. Clark, Fault Diagnosis in Dynamic Systems: Theory and Application. Upper Saddle River, NJ, USA: Prentice Hall, 1989. 